\section{Fatores associados a não realização da mamografia e do exame clínico das mamas: um estudo de base populacional em Campinas, São Paulo, Brasil}

\author{
Factors associated with lack of mammograms \\ and clinical breast examination by women: \\ a population-based study in Campinas, \\ São Paulo State, Brazil
}

Vivian Mae Schmidt Lima Amorim 1 Marilisa Berti de Azevedo Barros 1 Chester Luiz Galvão César 2

Luana Carandina ${ }^{3}$

Moisés Goldbaum 4

\footnotetext{
${ }^{1}$ Faculdade de Ciências Médicas, Universidade Estadual de Campinas, Campinas, Brasil. 2 Faculdade de Saúde Pública, Universidade de São Paulo, São Paulo, Brasil. 3 Faculdade de Medicina, Universidade Estadual Paulista, Botucatu, Brasil. 4 Faculdade de Medicina, Universidade de São Paulo, São Paulo, Brasil.

Correspondência V. M. S. L. Amorim Departamento de Medicina Preventiva e Social Faculdade de Ciências Médicas, Universidade Estadual de Campinas. C. P. 6111, Campinas, SP 13083-970, Brasil. vivianmae@dglnet.com.br
}

\begin{abstract}
This study analyzes the prevalence of mammograms and clinical breast examination according to socioeconomic, demographic, and health-related behavioral variables in women 40 years or older in Campinas, São Paulo State, Brazil. This was a cross-sectional population-based study $(N=290)$. Based on multivariate analysis, factors associated with not having a mammogram in the previous two years were: age ( $\geq 70$ years), racelethnicity (black or mixed-race), and low per capita family income. Lack of clinical breast examination in the previous year was associated with: marital status (single/widow/without partner) and low income. The Unified National Health System (SUS) performed 28.8\% of reported mammograms and $38.2 \%$ of clinical breast examinations. According to the findings, lack of breast cancer screening is associated with social and racial inequity, and reduction in breast cancer mortality requires early detection of the disease, which in turn demands that health care facilities implement strategies to expand coverage of preventive practices, especially for more vulnerable social groups.
\end{abstract}

Mammography; Breast Neoplasm; Women's Health

\section{Introdução}

Na maioria dos países, o câncer de mama tem apresentado incidências elevadas e crescentes. Dos casos registrados no mundo, $43 \%$ estão em países em desenvolvimento, chamando a atenção para a necessidade de implementação de ações de promoção da saúde, de prevenção e tratamento desse câncer também nas regiões economicamente menos favorecidas 1,2,3.

$\mathrm{O}$ câncer de mama é relativamente raro antes dos 35 anos, mas, acima desta idade sua incidência cresce rápida e progressivamente 4 . Mais de 85\% dos casos ocorrem após os 40 anos, alcançando o pico de incidência na faixa dos 65 a 70 anos 5 . A neoplasia de mama vem sendo um alvo privilegiado dos estudos da comunidade científica, o que possibilitou a identificação de vários fatores causais e um conhecimento maior sobre a sua história natural ${ }^{3}$. Fatores genéticos (história familiar), reprodutivos e hormonais (menarca precoce, menopausa tardia, idade avançada para a primeira gravidez, nuliparidade e lactação, uso de contraceptivos orais e terapia de reposição hormonal), e exposições ambientais (radiações ionizantes) foram identificados como fatores de risco para o surgimento do câncer de mama. Hábitos relacionados ao estilo de vida, como dieta rica em gordura, consumo elevado de álcool, baixa ingestão de vitaminas antioxidantes e sedentarismo também são apontados como positivamente associados a essa neoplasia 1,3. 
A prevenção primária, constituída de ações desencadeadas para evitar o surgimento da doença, é factível para alguns dos fatores de risco do câncer de mama, como o uso de contraceptivos orais e de terapia hormonal, obesidade pós-menopausa, exposição a radiações ionizantes e fatores relacionados ao estilo de vida 3 . Para muitos dos outros fatores não poderiam ser desenvolvidas estratégias específicas de prevenção. Para o controle do câncer de mama é fundamental a adoção de práticas de prevenção secundária, que compreendem o desencadeamento de ações de rastreamento para detecção precoce, evitando a progressão do câncer para estágios mais avançados e aumentando a probabilidade da cura 1,3.

Para o rastreamento da doença, visando à detecção precoce do tumor, são recomendadas três práticas preventivas: o auto-exame mensal das mamas; o exame clínico anual das mamas realizado por profissional de saúde; e a mamografia, que consiste em exame radiológico das mamas, capaz de identificar lesões subclínicas 1,6.

Considerando a necessidade da detecção precoce do câncer de mama e a incidência crescente com a idade, este estudo teve como objetivo analisar a realização das práticas preventivas para esta doença entre mulheres de 40 anos ou mais de idade, residentes na cidade de Campinas, São Paulo, Brasil, e identificar os fatores associados a não realização da mamografia e do exame clínico das mamas com a periodicidade recomendada pelo Ministério da Saúde.

\section{Métodos}

\section{População de estudo}

Este estudo transversal de base populacional utilizou dados do Inquérito Multicêntrico de Saúde no Estado de São Paulo (ISA-SP), realizado por pesquisadores das universidades públicas paulistas (Universidade de São Paulo [USP], Universidade Estadual Paulista [UNESP] e Universidade Estadual de Campinas [UNICAMP]) em parceria com a Secretaria Estadual de Saúde de São Paulo, em alguns municípios do estado, no período de 2001 a 2002 7. A pesquisa teve como base uma amostra probabilística, estratificada, por conglomerados e obtida em dois estágios de seleção ${ }^{8}$.

No primeiro, os setores censitários das áreas selecionadas foram agrupados em três estratos, segundo o porcentual de chefes de família com nível universitário: menos de 5\%, de 5\% a 25\% e $25 \%$ ou mais, visando a aumentar a probabilidade de que indivíduos pertencentes aos estratos de maior nível sócio-econômico da população fossem incluídos na amostra. Foram seleciona- dos dez setores de cada estrato, de cada área estudada, com probabilidade proporcional ao tamanho, por meio de sorteio sistemático aplicado à relação dos setores ordenados segundo tipo de setor dentro de cada estrato.

No segundo estágio da amostragem foram escolhidos os domicílios, também por meio de sorteio sistemático aplicado às relações de domicílios existentes em cada setor sorteado, tendo sido selecionados de 122 a 157 domicílios por setor, dependendo da área do estudo 8 .

Foram definidos domínios de idade e sexo em relação aos quais era desejável se obter um tamanho mínimo de amostra. Esses domínios foram: < 1 ano, 1 a 11 anos, 12 a 19 masculino, 12 a 19 feminino, 20 a 59 masculino, 20 a 59 feminino, 60 e mais masculino e 60 e mais feminino. Estimou-se um tamanho mínimo de amostra de 192 indivíduos para cada domínio de idade e sexo previamente definido, tendo como base a estimativa de prevalência de $50 \%$ com nível de $95 \%$ de confiança, erro máximo de 0,10 e efeito do delineamento (deff) igual a 2. Considerando-se os possíveis efeitos da não resposta (casa fechada e recusa) em torno de $20 \%$, o tamanho da amostra foi corrigido para 250 indivíduos em cada domínio 8 .

Foram sorteadas subamostras de domicílios, nas quais as pessoas dos distintos grupos de sexo/idade deveriam ser entrevistadas. A definição das pessoas que deveriam ser entrevistadas em cada domicílio obedeceu à probabilidade de se encontrar 250 pessoas de cada domínio de sexo e idade ${ }^{8}$.

As informações foram obtidas por entrevistadores treinados, por meio de questionário estruturado em 19 blocos temáticos, com a maioria das questões fechadas. As questões de interesse específico para este trabalho referem-se a características sócio-econômicas da entrevistada e do(a) chefe da família, realização de exames preventivos, presença de doenças crônicas auto-referidas, deficiência física, comportamentos relacionados à saúde, saúde emocional e autoavaliação da saúde.

Para o presente estudo foram utilizados dados referentes a todas as mulheres com 40 anos ou mais, não institucionalizadas, residentes na área urbana de Campinas, com respostas válidas para a realização de exame clínico das mamas e mamografia $(n=290)$.

As variáveis dependentes foram, portanto:

- Exame clínico das mamas realizado por profissional de saúde no ano prévio;

- Mamografia realizada nos dois anos que antecederam a entrevista.

As variáveis independentes analisadas foram: 
- Sócio-econômicas e demográficas: idade, cor/raça (auto-referida), naturalidade, situação conjugal, escolaridade, renda familiar mensal per capita, situação ocupacional, religião, número de pessoas no domicílio e posse de bens duráveis. Para o cálculo da renda familiar per capita foram considerados os valores dos salários mínimos vigentes no país por ocasião da realização do inquérito: salário mínimo de $\mathrm{R} \$ 151,00$ (março a abril de 2001), $\mathrm{R} \$ 180,00$ (maio de 2001 a março de 2002) e de $R \$ 200,00$ (abril a setembro de 2002).

- Comportamentos relacionados à saúde: prática de atividade física em contexto de lazer pelo menos uma vez por semana e situação tabágica: fumante e não-fumante (incluindo quem nunca fumou e os ex-fumantes).

- Relacionadas ao estado de saúde: número de doenças crônicas referidas, presença de limitação física, transtorno mental comum avaliado pelo Self Reporting Questionnaire (SRQ-20), presença de obesidade utilizando-se o índice de massa corporal $\left(\mathrm{IMC}=\mathrm{kg} / \mathrm{m}^{2}\right)$ calculado com base no peso e altura auto-referidos. As mulheres com IMC $\geq 25$ foram classificadas como tendo sobrepeso ou obesidade. Para as mulheres com 60 anos ou mais de idade foi analisada a autoavaliação da saúde que havia sido coletada somente para este grupo etário.

- Outras práticas preventivas: realização do exame de Papanicolaou nos últimos três anos para as mulheres entre 40 e 59 anos e, ao menos uma vez na vida, para aquelas com 60 anos ou mais.

\section{Análise dos dados}

Os dados do inquérito foram digitados em banco elaborado com o programa Epi Info 6.04b (Centers for Disease Control and Prevention, Atlanta, Estados Unidos) e submetido à análise de consistência. Para as análises estatísticas realizadas neste estudo foi utilizado o programa Stata 7.0 (Stata Corp., College Station, Estados Unidos) que possibilita considerar as variáveis do plano complexo de amostragem: estratos, conglomerados e ponderações. As análises bivariadas incluíram estimativas de prevalência e intervalos de 95\% confiança (IC95\%) da "não realização do exame clínico das mamas" e da "não realização da mamografia" segundo variáveis sócio-econômicas, de comportamentos relacionados à saúde e estado de saúde. As razões de prevalência ajustadas por idade e o modelo final foram realizados por meio de regressão de Poisson com variância robusta. Para entrada no modelo múltiplo foram consideradas as variáveis independentes sóciodemográficas, de comportamentos relacionados à saúde e de estado de saúde (Tabelas 1 e 2) que apresentaram valor de $\mathrm{p}$ inferior a 0,20 na análise bivariada, tendo permanecido no modelo final as variáveis que apresentavam $\mathrm{p}<0,05$. Não foi incluída no modelo final a variável auto-avaliação da saúde por estar disponível apenas para mulheres com 60 anos ou mais.

$\mathrm{O}$ projeto de pesquisa que resultou neste artigo foi aprovado pela Comissão de Ética da Faculdade de Ciências Médicas da UNICAMP sob parecer 369/2000.

\section{Resultados}

Dentre as 290 mulheres de 40 anos ou mais com respostas válidas para exame clínico das mamas e mamografia, predominaram as de 60 anos ou mais $(71,7 \%)$, de cor auto-referida como branca $(81,9 \%)$, sem cônjuge $(57,7 \%)$ e com até quatro anos de escolaridade $(64,4 \%)$.

Verificou-se no presente estudo que 50,8\% (IC95\%: 40,6-60,9) das mulheres de 40 anos ou mais, residentes no Município de Campinas, não haviam realizado mamografia nos dois anos que antecederam a entrevista (Tabela 1), sendo que $42,5 \%$ (IC95\%: 33,6-51,9) nunca haviam feito o exame e 8,3\% haviam-no realizado há mais de 2 anos; $38,2 \%$ (29,4-47,9\%) das mulheres não realizaram o exame clínico das mamas no ano prévio à entrevista.

Na Tabela 1, observa-se que a não realização do exame clínico das mamas e a não realização da mamografia foram significativamente mais prevalentes nas mulheres com 70 anos ou mais, de cor preta/parda e com renda familiar per capita menor ou igual a 5 salários mínimos. Nas que vivem sem companheiro e com escolaridade de até quatro anos, as associações observadas com a não realização do exame clínico deixaram de ser significativas após ajuste por idade.

Dos exames de mamografia realizados, $28,8 \%$ foram financiados pelo Sistema Único de Saúde (SUS) e $71,2 \%$ por serviços privados ou particulares. Quanto ao exame clínico das mamas, os serviços do SUS foram responsáveis pela realização de $38,1 \%$ e os serviços privados por $61,9 \%$ (dados não apresentados em tabela).

Observou-se em relação à morbidade e aos comportamentos relacionados à saúde (Tabela 2) que houve associação estatística significante apenas entre exame clínico das mamas e atividade física, apresentando maior prevalência de não realização as mulheres que não praticavam atividade física em contexto de lazer.

Os resultados da análise de regressão múltipla de Poisson (Tabela 3) apontam que a não realização do exame clínico das mamas foi significativamente mais freqüente nas mulheres que 
Prevalência de não realização da mamografia nos últimos dois anos e do exame clínico anual das mamas segundo variáveis sócio-econômicas e demográficas em mulheres de 40 anos ou mais. Campinas, São Paulo, Brasil, 2001-2002.

\begin{tabular}{|c|c|c|c|c|c|}
\hline \multirow[t]{2}{*}{ Variáveis } & \multirow[t]{2}{*}{$\mathbf{N}$} & \multicolumn{2}{|c|}{ Exame clínico } & \multicolumn{2}{|c|}{ Mamografia } \\
\hline & & $\begin{array}{l}\text { Prevalência (\%) } \\
\text { [valor de p *] }\end{array}$ & $\begin{array}{c}\text { Razão de } \\
\text { prevalências } \\
\text { ajustadas } \\
\text { pela idade }\end{array}$ & $\begin{array}{c}\text { Prevalência (\%) } \\
\text { [valor de p *] }\end{array}$ & $\begin{array}{c}\text { Razão de } \\
\text { prevalências } \\
\text { ajustadas } \\
\text { pela idade }\end{array}$ \\
\hline Idade (em anos) ** & & {$[0,0474]$} & & {$[0,0564]$} & \\
\hline $40-59$ & 82 & 34,1 & 1 & 48,1 & 1 \\
\hline $60-69$ & 107 & 38,3 & $1,12(0,71-1,76)$ & 46,5 & $0,89(0,56-1,40)$ \\
\hline 70 ou mais & 101 & 56,5 & $1,65(1,04-2,63)$ & 67,7 & $1,47(1,10-1,95)$ \\
\hline Total & 290 & 38,2 & & 50,8 & \\
\hline Raça/cor *** (auto-referida) & & {$[0,0064]$} & & {$[0,0039]$} & \\
\hline Branca & 236 & 32,3 & 1 & 45,8 & 1 \\
\hline Preta/parda & 52 & 58,6 & $1,88(1,25-2,82)$ & 71,7 & $1,59(1,20-2,10)$ \\
\hline Naturalidade & & {$[0,7931]$} & & {$[0,3002]$} & \\
\hline São Paulo & 188 & 37,8 & 1 & 47,2 & 1 \\
\hline Outros estados & 101 & 38,6 & $1,10(0,75-1,61)$ & 55,2 & $1,23(0,89-1,69)$ \\
\hline Situação conjugal & & {$[0,0416]$} & & {$[0,1236]$} & \\
\hline Com companheiro & 139 & 31,9 & 1 & 44,1 & 1 \\
\hline Sem companheiro & 151 & 46,8 & $1,34(0,92-1,97)$ & 59,9 & $1,29(0,84-1,97)$ \\
\hline Escolaridade (em anos) & & {$[0,0360]$} & & {$[0,1496]$} & \\
\hline 5 ou mais & 103 & 28,1 & 1 & 43,3 & 1 \\
\hline $0-4$ & 186 & 47,9 & $1,60(0,94-2,73)$ & 58,0 & $1,30(0,85-1,99)$ \\
\hline Renda familiar per capita (em salários mínimos) & & {$[0,0004]$} & & {$[0,0061]$} & \\
\hline$>5$ & 53 & 13,0 & 1 & 23,3 & 1 \\
\hline$\leq 5$ & 237 & 44,4 & $3,36(1,60-7,07)$ & 57,5 & $2,44(1,11-5,35)$ \\
\hline Situação ocupacional & & {$[0,4580]$} & & {$[0,6114]$} & \\
\hline Trabalha & 74 & 34,9 & 1 & 48,2 & 1 \\
\hline Não trabalha & 218 & 41,0 & $0,99(0,58-1,69)$ & 53,0 & $1,01(0,65-1,55)$ \\
\hline Religião & & {$[0,2261]$} & & {$[0,7166]$} & \\
\hline Católica & 218 & 41,5 & $1,32(0,73-2,36)$ & 51,6 & $1,02(0,73-1,41)$ \\
\hline Outras & 72 & 30,0 & 1 & 48,8 & 1 \\
\hline
\end{tabular}

$\mathrm{N}$ = número de indivíduos na amostra não ponderada.

* Valor de $p$ do teste $\chi^{2}$;

** Razão de prevalência bruta (simples);

*** Excluídas 2 mulheres de cor/raça amarela.

referiram não ter companheiro e nas que pertenciam ao segmento de menor renda familiar per capita em relação às respectivas categorias de referência. Para a mamografia, os resultados da análise de regressão múltipla (Tabela 4) apontam que a não realização do exame foi significativamente mais freqüente nas mulheres com 70 anos ou mais, nas que se auto-referiram de cor preta ou parda e no subgrupo de menor renda familiar per capita, comparadas às respectivas categorias de referência.
A análise da associação entre práticas preventivas (Tabela 5) revelou maior prevalência de não realização do exame clínico das mamas nas mulheres que não estavam com rotina adequada em relação ao exame de Papanicolaou, ao autoexame das mamas e à mamografia. A não realização da mamografia foi mais elevada nas mulheres que não haviam realizado o exame clínico das mamas no ano que antecedeu a entrevista $\mathrm{e}$ que não estavam com a rotina adequada do Papanicolaou. 
Prevalência de não realização da mamografia nos últimos dois anos e do exame clínico anual das mamas segundo comportamentos relacionados à saúde e morbidade em mulheres com 40 anos ou mais. Campinas, São Paulo, Brasil, 2001-2002.

\begin{tabular}{|c|c|c|c|c|c|}
\hline \multirow[t]{2}{*}{ Variáveis } & \multirow[t]{2}{*}{$\mathbf{N}$} & \multicolumn{2}{|c|}{ Exame clínico } & \multicolumn{2}{|c|}{ Mamografia } \\
\hline & & $\begin{array}{c}\text { Prevalência (\%) } \\
\text { [valor de } p \text { *] }\end{array}$ & $\begin{array}{c}\text { Razão de } \\
\text { prevalências } \\
\text { ajustadas } \\
\text { pela idade }\end{array}$ & $\begin{array}{c}\text { Prevalência (\%) } \\
\text { [valor de p *] }\end{array}$ & $\begin{array}{c}\text { Razão de } \\
\text { prevalências } \\
\text { ajustadas } \\
\text { pela idade }\end{array}$ \\
\hline Tabagismo & & {$[0,1740]$} & & {$[0,6933]$} & \\
\hline Fumante & 41 & 28,6 & 1 & 53,7 & $1,77(0,78-1,77)$ \\
\hline Não/Ex-fumante & 249 & 41,5 & $1,31(0,69-2,47)$ & 49,8 & 1 \\
\hline Prática de exercícios físicos & & {$[0,0227]$} & & {$[0,0906]$} & \\
\hline $\operatorname{Sim}$ & 81 & 27,2 & 1 & 37,9 & 1 \\
\hline Não & 209 & 43,1 & $1,55(1,03-2,31)$ & 56,4 & $1,47(0,87-2,48)$ \\
\hline Sobrepeso & & {$[0,4045]$} & & {$[0,2347]$} & \\
\hline Sim & 138 & 32,9 & $1,20(0,78-1,84)$ & 43,0 & $1,21(0,89-1,65)$ \\
\hline Não & 117 & 39,2 & 1 & 52,2 & 1 \\
\hline Presença de doenças crônicas & & {$[0,4517]$} & & {$[0,6812]$} & \\
\hline $0-3$ & 187 & 36,2 & 1 & 50,8 & $1,13(0,83-1,54)$ \\
\hline 4 ou mais & 97 & 42,6 & $1,07(0,68-1,66)$ & 47,8 & 1 \\
\hline Limitação física referida & & {$[0,1026]$} & & {$[0,4180]$} & \\
\hline Não & 213 & 35,3 & 1 & 48,9 & 1 \\
\hline Sim & 77 & 49,8 & $1,25(0,81-1,94)$ & 58,3 & $1,09(0,69-1,70)$ \\
\hline Transtorno mental comum & & {$[0,5319]$} & & {$[0,8617]$} & \\
\hline Não & 204 & 36,3 & 1 & 49,3 & 1 \\
\hline Sim & 71 & 42,2 & $1,14(0,71-1,82)$ & 51,0 & $1,02(0,69-1,52)$ \\
\hline Percepção da saúde ** & & {$[0,0568]$} & & {$[0,0644]$} & \\
\hline Excelente/Muito boa & 59 & 37,4 & 1 & 45,9 & 1 \\
\hline Boa & 118 & 49,7 & $1,28(0,87-1,88)$ & 57,7 & $1,21(0,88-1,68)$ \\
\hline Ruim/Muito ruim & 23 & 65,3 & $1,61(0,99-2,61)$ & 71,3 & $1,44(0,98-2,12)$ \\
\hline
\end{tabular}

$\mathrm{N}$ = número de indivíduos na amostra não ponderada.

* Valor de $p$ no teste $\chi^{2}$ entre colchetes;

** Mulheres com 60 anos ou mais.

\section{Discussão}

O Inquérito Multicêntrico de Saúde no Estado de São Paulo (ISA-SP) 7 trabalhou com três áreas temáticas: estilo de vida, situação de saúde e o uso de serviços de saúde. Nesse último, está inserido o bloco temático sobre a realização de alguns exames preventivos, entre eles o exame clínico das mamas e a mamografia, que são objetos deste artigo.

Uma das limitações deste estudo é o fato da informação sobre a realização do exame clínico das mamas e da mamografia ter sido obtida por entrevista e, portanto, ser sujeita a viés de memória e de informação. A entrevistada pode ter se enganado quanto ao tempo decorrido da realização do último exame, ou ainda dizer que realizou os exames por considerar esta a resposta adequada e esperada. Várias pesquisas têm uti- lizado informação referida sobre a realização do exame clínico das mamas e da mamografia 2,9,10, sendo que, alguns estudos realizados nos Estados Unidos observaram uma alta correlação entre os dados referidos e os registrados em prontuários, apontando as entrevistas como um método confiável e menos dispendioso para a obtenção deste tipo de informação 11,12.

Outra limitação deste estudo é o fato do inquérito abranger uma temática ampla, sem detalhamento maior de questões relativas às práticas preventivas do câncer de mama: não foram levantadas informações sobre se as mulheres conheciam o resultado do exame realizado e que atitudes tinham em relação a este tipo de teste. $\mathrm{O}$ desenho transversal do estudo limita, também, a possibilidade de interpretar as associações encontradas como derivadas de relações causaefeito. 
Modelo de regressão múltipla de Poisson para a não realização do exame físico das mamas em mulheres com 40 anos ou mais. Campinas, São Paulo, Brasil, 2001-2002.

\begin{tabular}{|c|c|c|c|c|}
\hline Variáveis & $\mathbf{N}$ & Razão de prevalências * & IC95\% & Valor de $p$ \\
\hline \multicolumn{5}{|l|}{ Situação conjugal } \\
\hline Com companheiro & 139 & 1 & & \\
\hline Sem companheiro & 151 & 1,49 & $1,08-2,08$ & 0,017 \\
\hline \multicolumn{5}{|c|}{ Renda familiar per capita (em salários mínimos) } \\
\hline$>5$ & 53 & 1 & & \\
\hline$\leq 5$ & 237 & 3,18 & $1,48-6,84$ & 0,004 \\
\hline
\end{tabular}

* Ajustada pelo modelo de Poisson para as demais variáveis do modelo.

Modelo de regressão múltipla de Poisson para a não realização da mamografia em mulheres com 40 anos ou mais. Campinas, São Paulo, Brasil, $2001-2002$.

\begin{tabular}{|c|c|c|c|c|}
\hline Variáveis & $\mathbf{N}$ & Razão de prevalências * & IC95\% & Valor de $p$ \\
\hline \multicolumn{5}{|l|}{ Idade (anos) } \\
\hline $40-59$ & 82 & 1,06 & $0,74-1,51$ & 0,735 \\
\hline $60-69$ & 107 & 1 & & \\
\hline 70 ou + & 101 & 1,48 & $1,18-1,86$ & 0,001 \\
\hline \multicolumn{5}{|l|}{ Raça/Cor } \\
\hline Branca & 236 & 1 & & \\
\hline Negra/Parda & 52 & 1,39 & $1,03-1,86$ & 0,028 \\
\hline \multicolumn{5}{|c|}{ Renda familiar per capita (em salários mínimos) } \\
\hline$>5$ & 53 & 1 & & \\
\hline$\leq 5$ & 237 & 2,26 & $1,00-5,09$ & 0,049 \\
\hline
\end{tabular}

* Ajustada pelo modelo de Poisson para as demais variáveis do modelo.

A escolha da faixa etária estudada - 40 anos ou mais - considerou os dados nacionais e as informações do município que apontam o aumento da incidência do câncer de mama a partir dos 40 anos de idade 5,13,14.

A presente pesquisa permitiu verificar que 38,2\% (IC95\%: 29,4-47,9\%) das mulheres com 40 anos ou mais residentes em Campinas não haviam feito exame clínico das mamas no ano prévio, e 50,8\% não tinham realizado mamografia nos dois anos que antecederam a entrevista. Para as mulheres da faixa etária de 40 a 69 anos, as prevalências da não realização do exame clínico das mamas $(36,2 \%)$ e da mamografia $(47,9 \%)$ foram maiores que as encontradas na Cidade de Pelotas onde foram verificadas prevalências de $18,6 \%$ e $31,1 \%$, respectivamente 15 . As prevalências de não realização do exame clínico das mamas e da mamografia em Campinas também se apresentaram maiores que as observadas nas cidades de Belo Horizonte (Minas Gerais), Vitória (Espírito Santo), Florianópolis (Santa Catarina) e Porto Alegre (Rio Grande do Sul), segundo os dados obtidos no inquérito realizado pelo Instituto Nacional de Câncer (INCA) 16. Observou-se ainda que, no tocante à realização da mamografia, $42,5 \%(33,8-52,3 \%)$ das mulheres com 40 anos ou mais nunca tinham feito este exame, achado que é semelhante ao encontrado para a população brasileira pelo Inquérito Mundial de Saúde, realizado no Brasil em 2003, que foi de 45,9\% 17. Considerando a estrutura e a organização da rede de serviço de saúde do Município de Campinas, a cobertura observada dos exames preventivos para o câncer de mama revelou-se bem inferior à esperada.

O presente estudo revelou que a não realização do exame clínico das mamas e da mamografia 
Prevalência de não realização da mamografia nos últimos dois anos e do exame físico das mamas segundo outras práticas preventivas e de cuidado à saúde, em mulheres de 40 anos ou mais. Campinas, São Paulo, Brasil, 2001-2002.

\begin{tabular}{|c|c|c|c|c|c|}
\hline \multirow[t]{2}{*}{ Variáveis } & \multirow[t]{2}{*}{$\mathbf{N}$} & \multicolumn{2}{|c|}{ Exame clínico } & \multicolumn{2}{|c|}{ Mamografia } \\
\hline & & $\begin{array}{l}\text { Prevalência (\%) } \\
\text { [valor de p *] }\end{array}$ & $\begin{array}{c}\text { Razão de } \\
\text { prevalências } \\
\text { ajustadas } \\
\text { pela idade }\end{array}$ & $\begin{array}{l}\text { Prevalência (\%) } \\
\left.\text { [valor de p }{ }^{\star}\right]\end{array}$ & $\begin{array}{c}\text { Razão de } \\
\text { prevalências } \\
\text { ajustadas } \\
\text { pela idade }\end{array}$ \\
\hline \multicolumn{6}{|l|}{ Auto-exame das mamas } \\
\hline (independente da freqüência) & & {$[0,0093]$} & & {$[0,2946] * \star$} & \\
\hline Sim & 204 & 32,2 & 1 & 48,4 & 1 \\
\hline Não & 86 & 54,3 & $1,64(1,14-2,36)$ & 57,2 & $1,16(0,85-1,59)$ \\
\hline Exame clínico das mamas (no último ano) & & & & {$[0,0000]$} & \\
\hline Sim & 164 & & & 26,8 & 1 \\
\hline Não & 126 & & & 89,6 & $3,30(2,17-5,02)$ \\
\hline Mamografia (nos últimos 2 anos) & & {$[0,0000]$} & & & \\
\hline Sim & 134 & 8,1 & 1 & & \\
\hline Não & 156 & 67,4 & $8,15(3,87-17,14)$ & & \\
\hline Exame de citologia oncótica & & {$[0,0000]$} & & {$[0,0000]$} & \\
\hline Adequada & 251 & 28,6 & 1 & 42,2 & 1 \\
\hline Não adequada & 39 & 86,2 & $3,16(2,04-4,90)$ & 93,8 & $2,24(1,68-3,00)$ \\
\hline
\end{tabular}

é maior nas mulheres mais idosas, resultado que é semelhante aos observados por outros estudos 2,18,19. Ressalte-se que os avanços tecnológicos e sociais ocorridos nas últimas décadas ao propiciarem o aumento da expectativa de vida, que se acompanha de elevação da prevalência de doenças crônicas, tendem a aumentar a demanda dos idosos por serviços de saúde. Se o atendimento às doenças crônicas é feito com uma abordagem de cuidado integral, as oportunidades de atenção podem ser utilizadas para a realização de práticas preventivas incluindo as do câncer de mama.

A menor prevalência da não realização da mamografia pelas mulheres auto-referidas de cor preta ou parda persistiu no modelo final após ajuste por idade e renda per capita, apontando uma possível desigualdade racial no acesso a este exame. Outros estudos brasileiros têm relatado menor acesso das mulheres negras aos exames de prevenção do câncer de mama 17,20, de colo de útero 21,22 e a outros serviços de assistência ao pré-natal e parto 23.

Também foi observada menor prevalência da não realização do exame clínico das mamas por mulheres que relataram não ter companheiro, mesmo com ajuste por idade e renda. Estudo realizado na cidade de São Leopoldo (Rio Grande do Sul), também encontrou menor prevalência na realização do exame clínico das mamas em mulheres não casadas 18 . É possível aventar que a percepção da necessidade de consulta ginecológica para problemas originados com a vida sexual ativa e para propostas de contracepção, e não a uma perspectiva mais ampla de cuidado com a saúde, levaria mulheres sem companheiro a demandarem menos os serviços ginecológicos e, portanto, a terem menor acesso ao exame clínico das mamas. Mas, outro estudo brasileiro identificou que as mulheres pertencentes a famílias chefiadas por homens apresentam maior chance de utilização de serviços preventivos e de acompanhamento, levantando como hipótese explicativa que restrições sociais e econômicas limitariam o consumo de serviços de saúde pelas mulheres que são chefes de família 24

$\mathrm{O}$ presente estudo apontou que mulheres do segmento de menor nível sócio-econômico, avaliado pela renda familiar per capita, tiveram maior prevalência de não realização dos exames analisados. Em relação à mamografia esse achado é semelhante ao encontrado na Pesquisa Nacional por Amostra de Domicílios (PNAD), realizada em 2003, em que foi observado que a prevalência da não realização do exame aumen- 
tava quanto menor a renda familiar per capita 25 . Estudos nacionais $16,17,18,26$ e internacionais 2,9,27,28 observaram também menor prevalência de realização do exame clínico das mamas e da mamografia em mulheres de menor nível sócioeconômico, avaliado pela escolaridade ou por outros indicadores. Esses resultados apontam o grau de iniqüidade de acesso ao serviço ainda presente no Município de Campinas e a necessidade de ampliação de cobertura das práticas preventivas analisadas nos segmentos sociais mais carentes.

Outro achado relevante do presente estudo é que somente $28,8 \%$ das mamografias realizadas foram financiadas pelo SUS e apenas $38,2 \%$ das mulheres que realizaram o exame clínico anual das mamas o fizeram em serviços do SUS, enquanto que o inquérito realizado pelo INCA 16 havia apontado que os serviços do SUS eram responsáveis por $48,7 \%$ dos exames de rastreamento para o câncer de mama na cidade de São Paulo. Pelo alto custo do exame, o Ministério da Saúde recomenda que a adoção da mamografia, como estratégia de rastreamento populacional, seja destinada somente às mulheres de 50 a 69 anos, e fora desta faixa etária, apenas para os casos em que o exame clínico das mamas apresentar alterações 6 . Essa restrição poderia ser a causa da maior proporção de mamografias realizadas em serviços não vinculados ao SUS, que teriam maior flexibilidade para a indicação e a realização do exame. Também tem sido observado em Campinas, que outras atividades relacionadas à saúde da mulher têm apresentado grande parcela de atendimentos realizados por planos e convênios de saúde que respondem por $46,8 \%$ do atendimento pré-natal do município 29 e 56,8\% da realização do exame de citologia oncótica ${ }^{21}$.
Outros estudos brasileiros mostram que a utilização de serviços preventivos de saúde é mais freqüente entre aqueles que possuem plano privado de saúde 30 .

Observou-se a concomitância de não realização das práticas preventivas, incluindo: exame clínico das mamas, auto-exame mensal das mamas, citologia oncótica e mamografia. Esse fato é esperado visto que o exame clínico das mamas, a solicitação da mamografia e a realização do exame de Papanicolaou fazem parte do atendimento prestado na consulta ginecológica, e que, na ausência desta consulta, a realização de todos estes procedimentos fica comprometida. Pressupõe-se ainda que as mulheres que procuram mais os serviços de saúde para a consulta ginecológica também estariam mais expostas a estímulos e orientação para o auto-exame das mamas, visto que a orientação para esta prática também deve ser parte integrante da consulta ginecológica 6 .

Pode-se concluir que os achados deste estudo apontam a existência de significativas desigualdades sócio-econômicas e raciais quanto ao acesso às práticas preventivas para o câncer de mama, reforçando a necessidade de intervenções que visem à promoção da eqüidade. O exame clínico das mamas e a mamografia são importantes práticas de detecção precoce do câncer de mama, cujo acesso precisa ser assegurado pelos serviços de saúde, pois contribuem para a redução da mortalidade por esta neoplasia. Muitos são os desafios a serem enfrentados pelos gestores das políticas de saúde na implementação de estratégias que reorientem os serviços na perspectiva da atenção integral à saúde das mulheres e com foco especial para as mais vulneráveis à baixa cobertura de práticas preventivas.

\section{Resumo}

O objetivo deste estudo é analisar a prevalência da não realização do exame clínico das mamas e da mamografia segundo variáveis sócio-econômicas, demográficas e de comportamentos relacionados à saúde, em mulheres com 40 anos ou mais, residentes na cidade de Campinas, São Paulo, Brasil. O estudo foi do tipo transversal, de base populacional em uma amostra de 290 mulheres. Os fatores associados à não realização da mamografia, encontrados na análise multivariada, foram: ter 70 anos ou mais, ser de raça/cor negra e pertencer ao segmento de menor renda familiar per capita; e para a não realização do exame clínico das mamas foram: não ter companheiro e pertencer ao segmento de menor renda familiar per capita. O SUS foi responsável pela realização de $28,8 \%$ das mamografias e 38,2\% dos exames clínicos das mamas. Verificou-se que a não realização dos exames preventivos para o câncer de mama está associada à existência de desigualdade racial e social, apontando para a necessidade de implementação de estratégias para a ampliação da cobertura das práticas preventivas para o câncer de mama, especialmente para os segmentos sociais mais vulneráveis.

Mamografia; Neoplasia da Mama; Saúde da Mulher 


\section{Colaboradores}

V. M. S. L. Amorim realizou a proposta do artigo, a revisão da literatura, análise dos dados e a redação. M. B. A. Barros orientou a proposta do artigo, a análise dos dados e a redação. M. B. A. Barros, C. L. G. César, L. Carandina, M. Goldbaum desenvolveram o projeto Inquérito Multicêntrico de Saúde no Estado de São Pau$l o$, elaboraram os instrumentos, coordenaram a pesquisa de campo e contribuíram na revisão do artigo.

\section{Agradecimentos}

À FAPESP (Fundação de Amparo à Pesquisa do Estado de São Paulo) - Programa de Políticas Públicas, processo no. 88/14099-7, e à Secretaria Estadual de Saúde de São Paulo, pelo financiamento do Inquérito Multicêntrico de Saúde do Estado de São Paulo. À Secretaria de Vigilância em Saúde do Ministério da Saúde, pelo suporte à análise por meio do Centro Colaborador em Análise de Situação de Saúde do Departamento de Medicina Preventiva e Social da Faculdade de Ciências Médicas, Universidade Estadual de Campinas.

\section{Referências}

1. Robles SC, Galanis E. Breast cancer in Latin America and the Caribbean. Rev Panam Salud Pública 2000; 11:178-85.

2. Siahpush M, Singh GK. Sociodemographic variations in breast cancer screening behavior among Australian women: results from the 1995 National Health Survey. Prev Med 2002; 35:174-80.

3. Adams EK, Florence CS, Thorpe KE, Becker ER, Joski PJ. Preventive care: female cancer screening 1996-2000. Am J Prev Med 2003; 25:301-7.

4. Almeida OJ, Zeferino LC, Teixeira LC. Mamografia de rastreamento em mulheres idosas. Femina 1999; 27:627-9.

5. Finotti MCCF, Freitas Junior R. A importância da mamografia no climatério. Femina 1998; 26:487-91.

6. Instituto Nacional de Câncer. Controle do câncer de mama. Documento de consenso. Rio de Janeiro: Instituto Nacional de Câncer; 2004.

7. César LGC, Carandina L, Alves MCGP, Barros MBA, Goldbaum M. Saúde e condição de vida em São Paulo. Inquérito Multicêntrico de Saúde no Estado de São Paulo - ISA-SP. São Paulo: Faculdade de Saúde Pública, Universidade de São Paulo; 2005.

8. Alves MCG. Plano de amostragem. In: César LGC, Carandina L, Alves MCGP, Barros MBA, Goldbaum M, organizadores. Saúde e condição de vida em São Paulo. Inquérito Multicêntrico de Saúde no Estado de São Paulo - ISA-SP. São Paulo: Faculdade de Saúde Pública, Universidade de São Paulo; 2005. p. 47-62.
9. Meissner HI, Breen N, Yabroff R. Whatever happened to clinical breast examination? Am J Prev Med 2003; 25:259-63.

10. Coughlin SS, Uhler R. Breast and cervical screening practices among Asian and Pacific Islander women in United States, 1994-1997. Cancer Epidemiol Biomarkers Prev 2000; 9:597-603.

11. Montano DE, Phillips WR. Cancer screening by primary care physicians: a comparison of rates obtained from physician self-report, patient survey, and chart audit. Am J Public Health 1995; 85: 795-800.

12. Caplan LS, McQueen DV, Qualters ML, Garret C, Calonge N. Validity of women's self-reports of cancer screening test utilization in a managed care population. Cancer Epidemiol Biomark Prev 2003; 12:1182-7.

13. Instituto Nacional de Câncer. Câncer no Brasil - dados dos registros de base populacional. http:// www.inca.gov.br (acessado 05/Ago/2005).

14. Secretaria Municipal de Saúde de Campinas/Universidade de Campinas. Mortalidade em Campinas. Boletim no 36 julho a dezembro de 2004. Mortalidade por câncer de mama e colo de útero. http://www.campinas.sp.gov.br/saúde (acessado 10/Fev/2006).

15. Sclowitz ML, Menezes AMB, Gigante DP, Tessaro S. Condutas na prevenção secundária do câncer de mama e fatores associados. Rev Saúde Pública 2005; 39:340-9. 
16. Instituto Nacional de Câncer. Inquérito domiciliar sobre comportamento de risco e morbidade referida de doenças e agravos não transmissíveis: Brasil, 15 capitais e Distrito Federal, 2002-2003. Rio de Janeiro: Instituto Nacional de Câncer; 2004.

17. Leal MC, Gama SGN, Frias P, Szwarcwald CL. Healthy lifestyles and access to periodic health exams among Brazilian women. Cad Saúde Pública 2005; 21 Suppl:S78-88.

18. Frazier EL, Jiles RB, Mayberry R. Use of screening mammography and clinical breast examinations among black, hispanic and white women. Prev Med 1996; 25:118-25.

19. Coughlin S, Uhler R. Breast and cervical screening pratices among Hispanic women in the United State and Puerto Rico, 1998-1999. Prev Med 2002; 34:242-51.

20. Dias-da-Costa JS, Olinto MTA, Bassani D, Marchionatti CRE, Bairros FS, Oliveira MLP, et al. Desigualdades na realização do exame clínico de mama em São Leopoldo, Rio Grande do Sul, Brasil. Cad Saúde Pública 2007; 23:1603-12.

21. AmorimVMSL, Barros MBA, César CLG, CarandinaL, Goldbaum M. Fatores associados à não realização do exame de Papanicolaou: um estudo de base populacional no Município de Campinas, São Paulo, Brasil. Cad Saúde Pública 2006; 22:2329-38.

22. Dias-da-Costa JS, Olinto MTA, Gigante DP, Menezes AMB, Macedo S, Borba AT, et al. Cobertura do exame citológico na Cidade de Pelotas, Rio Grande do Sul, Brasil. Cad Saúde Pública 2003; 19:191-7.

23. Travassos C, Viacava F, Pinheiros R, Brito A. Utilização dos serviços de saúde no Brasil: gênero, características familiares e condição social. Rev Panam Salud Pública 2002; 11:365-73.
24. Leal MC, Gama SGN, Cunha CB. Desigualdades raciais, sociodemográficas e na assistência ao prénatal e ao parto, 1999-2001. Rev Saúde Pública 2005; 39:100-7.

25. Lima-Costa MF, Matos DL. Prevalência e fatores associados à realização da mamografia na faixa etária de 50-69 anos: um estudo baseado na Pesquisa Nacional por Amostra de Domicílios (2003). Cad Saúde Pública 2007; 23:1665-73.

26. Novaes HMD, Braga PE, Schout D. Fatores associados à realização de exames preventivos para câncer nas mulheres brasileiras, PNAD 2003. Ciênc Saúde Coletiva 2006; 11:1023-35.

27. Abraido-Lanza AF, Chao MT, Gammon MD. Breast and cervical cancer screening among latinas and non latinas whites. Am J Public Health 2004; 94:1393-8.

28. Katz SJ, Zemencuk JK, Timothy P. Breast cancer screening in United States and Canada, 1994: socioeconomics gradients persit. Am J Public Health 2000; 90:799-803.

29. Almeida SDM, Barros MBA. Eqüidade e atenção à saúde da gestante em Campinas (SP), Brasil. Rev Panam Salud Pública 2005; 17:15-25.

30. Lima-Costa MF. Estilos de vida e uso de serviços preventivos de saúde entre adultos filiados ou não a plano privado de saúde (inquérito de saúde de Belo Horizonte). Ciênc Saúde Coletiva 2004; 9:857-64.

Recebido em 13/Set/2007

Versão final reapresentada em 16/Abr/2008 Aprovado em 18/Abr/2008 